\title{
Amygdala Inputs to the Prefrontal Cortex Elicit Heterosynaptic Suppression of Hippocampal Inputs
}

\author{
Hugo A. Tejeda ${ }^{1,2}$ and Patricio 0'Donnell ${ }^{1,3}$ \\ ${ }^{1}$ Department of Anatomy and Neurobiology, ${ }^{2}$ Program in Neuroscience/National Institutes of Health Graduate Partnership Program, and ${ }^{3}$ Department of \\ Psychiatry, University of Maryland School of Medicine, Baltimore, Maryland 21201
}

\begin{abstract}
Whereas cooperative communication between the hippocampus (HP) and prefrontal cortex (PFC) is critical for cognitive functions, an antagonistic relationship may exist between the basolateral amygdala (BLA) and PFC during emotional processing. As PFC neurons integrate information from converging excitatory BLA and HP inputs, we explored whether the ability of BLA inputs to evoke feedforward inhibition in the PFC affects converging HP synaptic inputs using in vivo intracellular recordings in anesthetized rats. BLA train stimulation decreased HP synaptic responses in the PFC in vivo. This effect was dependent on the timing of HP-evoked responses and the strength of BLA activation. BLA train stimulation also produced heterosynaptic suppression of responses from the amygdalo-piriform cortex, an associative temporal cortical structure. Heterosynaptic suppression was unidirectional as HP trains failed to modify BLA synaptic responses. These findings provide a mechanism by which BLA activation could decrease PFC neural activity and transiently attenuate the HP influence on PFC function.
\end{abstract}

Key words: amygdala; electrophysiology; in vivo intracellular recording; prefrontal cortex

\section{Introduction}

Finely tuned prefrontal cortical (PFC) function is critical for cognition, executive functions, and regulation of emotional processing (Bechara and Van Der Linden, 2005; Chudasama and Robbins, 2006; Townsend and Altshuler, 2012). The PFC role in cognitive and executive functions depends on interactions with other limbic and cortical structures, including the basolateral amygdala (BLA), hippocampus (HP), and associative cortices, and the manner inputs from these other structures are integrated in the PFC is likely to affect these functions. The BLA is critical for processing emotional and motivational information (Bechara et al., 2003; Phelps and LeDoux, 2005), and BLA-PFC interactions are required for decision making, behavioral control, and emotional processing (Floresco and Ghods-Sharifi, 2007; Churchwell et al., 2009; Kim et al., 2011b; Sotres-Bayon et al., 2012). On the other hand, communication between the HP and PFC is critical for cognition, and cognitive performance requires coordinated HP and PFC activity (Eichenbaum, 2000; Gordon, 2011). Thus,

\footnotetext{
Received March 1, 2014; revised Sept. 13, 2014; accepted Sept. 18, 2014.

Author contributions: P.O. and H.A.T. designed research; H.A.T. performed research; P.O. and H.A.T. analyzed data; P.O. and H.A.T. wrote the paper.

This work was supported by National Institutes of Health Grant R01 MH057683 to P.0. We thank Dr. Karl Deisseroth for the generous donation of the adeno-associated virus CaMKII-CHR2 construct for the optogenetics part of the study.

P.O. is currently employed and stockholder of Pfizer, Inc. The remaining author declares no competing financial interests.

Correspondence should be addressed to either of the following: Dr. Hugo A. Tejeda, National Institute of Drug Abuse, 251 Bayview Blvd, Room 05A311, Baltimore, MD 21224, E-mail: tejedah@nida.nih.gov; or Dr. Patricio O'Donnell, Neuroscience Research Unit, Pfizer, Inc, 610 Main Street, Cambridge, MA 02139, E-mail: patricio.odonnell@pfizer.com.

DOI:10.1523/JNEUROSCI.0837-14.2014

Copyright $\odot 2014$ the authors $\quad 0270-6474 / 14 / 3414365-10 \$ 15.00 / 0$
}

the manner in which PFC neurons integrate HP and BLA inputs is a key element in their role in higher-order behaviors.

BLA and PFC activity is often inversely correlated. Increases in BLA activity are associated with decreased PFC activity in humans (Kim et al., 2011a; Sripada et al., 2012; Townsend and Altshuler, 2012), and these opposing changes are principally observed during emotional processing, anxiety, and stress (Kim et al., 2011a; Veer et al., 2012). This inverse relationship has been suggested to reflect a "top-down" PFC regulation of the BLA, as cognitive control of emotional processing is associated with increased PFC activation and decreased amygdala activity (Townsend and Altshuler, 2012). In rodents, PFC stimulation suppresses BLA projection neuron activity (Rosenkranz and Grace, 2002; but see Likhtik et al., 2005). Conversely, BLA stimulation inhibits the majority of PFC neurons (Pérez-Jaranay and Vives, 1991; Ishikawa and Nakamura, 2003; Floresco and Tse, 2007; Dilgen et al., 2013) via a feedforward inhibition mechanism. Indeed, BLA stimulation enhances the activity of PFC GABAergic interneurons and evokes inhibitory synaptic responses in PFC pyramidal neurons (Dilgen et al., 2013). This interaction provides a framework whereby a "bottom-up" inhibition of the PFC by the BLA can occur in response to emotionally arousing stimuli via recruitment of feedforward inhibition.

It is possible that BLA-evoked feedforward inhibition affects PFC responses to other inputs. Here, we investigated whether BLA stimulation affects PFC responses to activation of inputs from the HP and the amygdalo-piriform cortex (AP), an associative complex in the temporal cortex. We used in vivo intracellular recordings with optogenetics to determine subthreshold synaptic interactions between BLA and HP inputs in PFC neurons, assessing whether strong BLA activation results in heterosynaptic suppression of HP-evoked synaptic responses. 

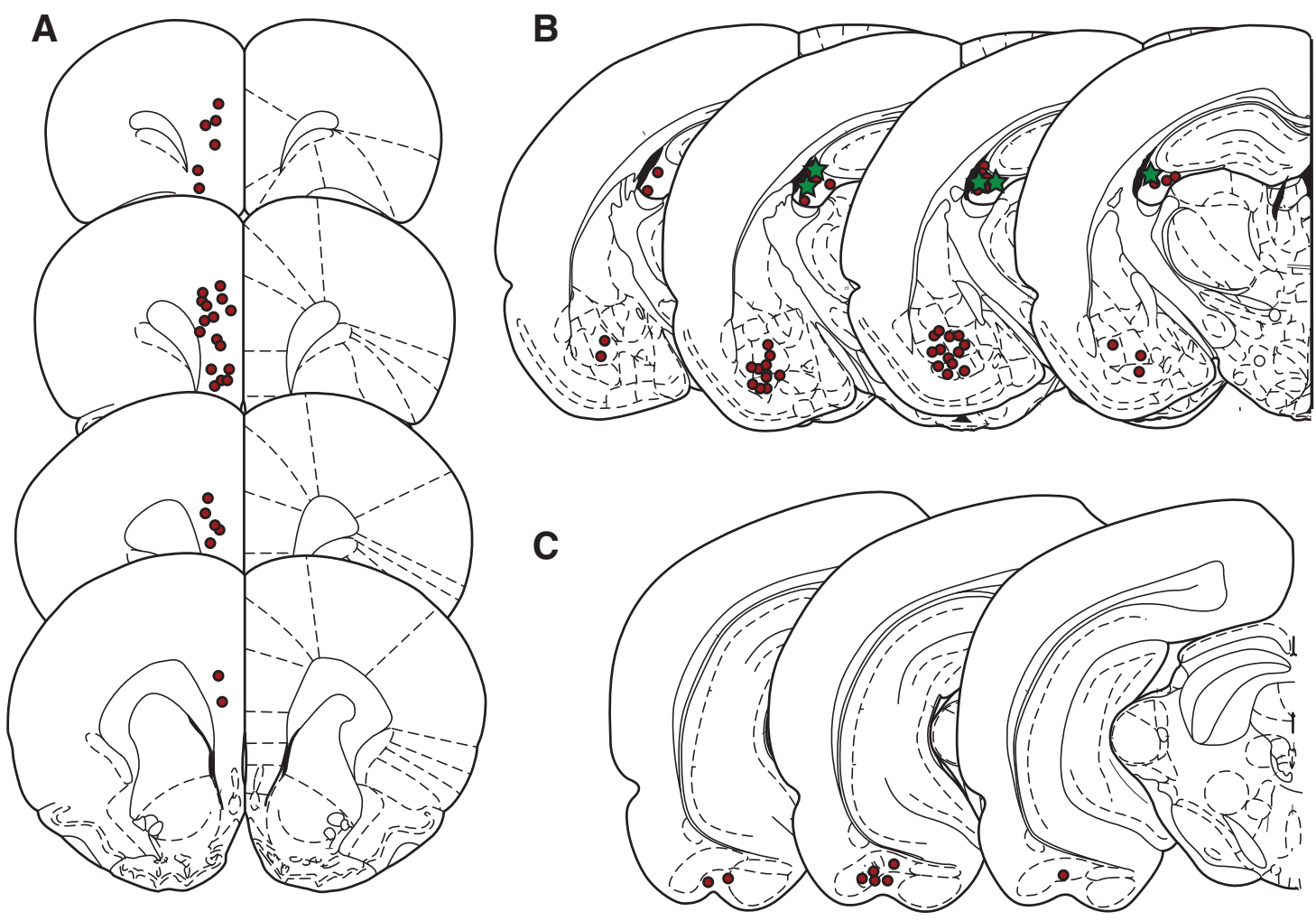

Figure 1. Recording sites in the medial PFC and stimulation sites in the BLA, fornix, and AP cortex. A, Location of neurobiotin-filled pyramidal neurons or histologically confirmed electrode placement in the medial PFC of rats (red circles). B, Stimulating electrode placements in the BLA and fornix (red circles). Green stars indicate location of optical fibers in the fornix. C, Stimulating electrode placements in the AP cortex (red circles).

\section{Materials and Methods}

Subjects. Male Long-Evans rats weighing 300-450 g (Charles River Laboratories) were kept in a temperature and humidity-controlled environment with lights on at 7:00 A.M. and lights off at 7 P.M., and with food and water available ad libitum. All experiments were conducted in accordance with guidelines published in the United States Public Health Service Guide for the Use and Care of Laboratory Animals, and all procedures were approved by the University of Maryland School of Medicine Institutional Animal Care and Use Committee.

In vivo intracellular electrophysiology. Rats were anesthetized with choral hydrate $(400 \mathrm{mg} / \mathrm{kg}$, i.p.). Anesthesia was maintained throughout the experiments using continuous choral hydrate $(24-30 \mathrm{mg} / \mathrm{kg} / \mathrm{h})$ via an intraperitoneal catheter. Body temperature was maintained at $\sim 37^{\circ} \mathrm{C}$ using a thermal probe-controlled heat pad (Fine Science Tools). Rats were fixed on a stereotaxic apparatus (Kopf Instruments). Concentric bipolar stimulating electrodes $(0.5 \mathrm{~mm}$ diameter, $0.5 \mathrm{~mm}$ pole separation; Rhodes Medical Instruments) were lowered into the left BLA [3.0 $\mathrm{mm}$ caudal to bregma (anteroposterior); 4.9-5.0 mm lateral to midline (mediolateral); $7.4 \mathrm{~mm}$ from dura (dorsoventral)] and left fornix [anteroposterior: $-3.0 \mathrm{~mm}$; mediolateral: $3.9-4.0 \mathrm{~mm}$; dorsoventral: -3.1 $\mathrm{mm}$ ]. Coordinates were based on the rat brain atlas of Paxinos and Watson (1998). Recording sharp microelectrodes were pulled from borosilicate glass (1 mm O.D.; World Precision Instruments) on a horizontal Flaming-Brown puller (Sutter Instruments). Sharp electrodes were filled with $2 \%$ Neurobiotin (Vector Laboratories) in $2 \mathrm{~m}$ potassium acetate. Microelectrodes were lowered into the superficial cortex using a hydraulic manipulator (Trent Wells), and electrode resistance was determined $(50-110 \mathrm{M} \Omega)$. Medial PFC coordinates were 2.8 to $3.4 \mathrm{~mm}$ anteroposterior, 0.6 to $1.0 \mathrm{~mm}$ mediolateral, and -2.5 to $5.5 \mathrm{~mm}$ dorsoventral from dura. Recordings were made in current clamp. Signals were passed through a headstage to a Neurodata Intracellular Amplifier (Cygnus) and continuously monitored on a digital oscilloscope (Fluke), a multimeter (Tektronix), and an audio monitor (Grass). Signals were digitized at 10
kHz using a Digidata (Molecular Devices) and acquired with Axoscope 9 software (Molecular Devices) for offline analysis.

Microelectrodes were advanced through the medial PFC while passing square current pulses $(0.2 \mathrm{nA} ; 100 \mathrm{~ms})$ to continuously monitor electrode resistance. After a neuron was impaled, baseline activity was recorded for $>3$ min before determining the input resistance of the neuron. Neurons included in this study had a resting membrane potential more negative than $-60 \mathrm{mV}$ and action potentials with amplitudes $\geq 40 \mathrm{mV}$ from threshold. To determine whether BLA activation influences hippocampal inputs to the medial PFC, we used a stimulation protocol wherein a baseline pulse was applied to the fornix (F1; $0.5 \mathrm{~ms}$ duration; $0.3-1.0 \mathrm{~mA}$ ), the fiber bundle that includes hippocampal fibers innervating the forebrain. After a $500 \mathrm{~ms}$ delay, a conditioning pulse train was applied to the BLA (BLA train $_{10} ; 10$ pulses; 10,20 , or $\left.50 \mathrm{~Hz}\right)$. A test pulse (F2; $0.5 \mathrm{~ms}$ duration; $0.3-1.0 \mathrm{~mA}$ ) was subsequently applied to the fornix at varying delays after the last $\mathrm{BLA}_{\text {train }}$ pulse $(50,100,150,300$, or $500 \mathrm{~ms})$ in either ascending or descending order. The order in which the F2 test pulse was applied was counterbalanced among cells, with approximately half being presented with $\mathrm{F} 2$ pulse in ascending delays and the other half being presented with descending F2 delays. The interstimulus intervals between the first and second fornix pulse (F1 and F2) ranged from 730 to $1180 \mathrm{~ms}, 1000$ to $1450 \mathrm{~ms}$, and 1450 to $1900 \mathrm{~ms}$ for the 50,20 , and $10 \mathrm{~Hz}$ stimulation protocols, respectively. We also determined whether a single conditioning pulse to the BLA would modify a subsequent F2 test pulse using the same protocol, with the exception that the BLA pulse train was replaced by a single pulse to the BLA (F1-BLA single $-\mathrm{F} 2)$. To determine whether alterations in F2 responses were due to short-term facilitation, we conducted the same stimulation protocol as described above but omitted BLA train stimulation (F1-NoBLA-F2). In some cases, the AP cortex was stimulated instead of the fornix to evaluate effects of BLA train stimulation on synaptic responses (AP1 and AP2) in another corticalPFC pathway. To determine whether HP inputs could in turn affect responses to BLA stimulation in the $\mathrm{PFC}$, we conducted similar experi- 
A
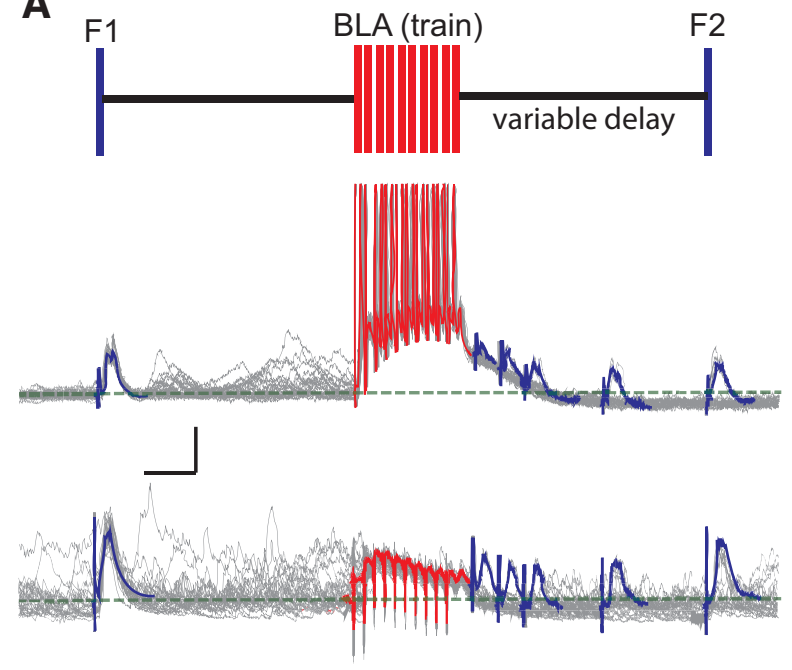

D F1

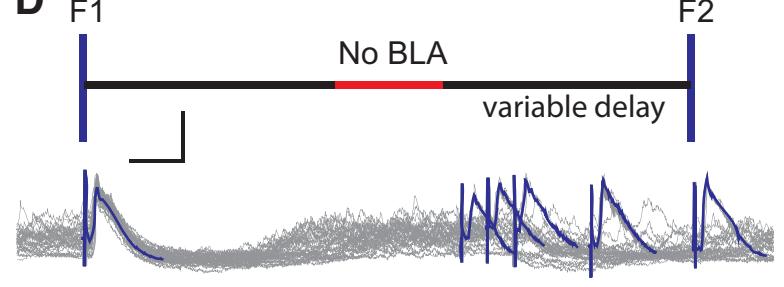

$\mathbf{F}$

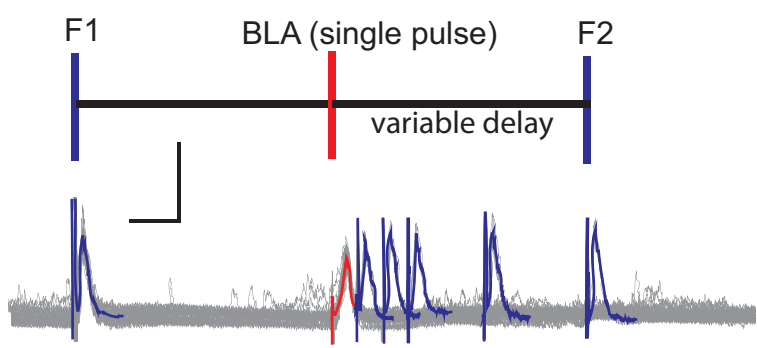

B

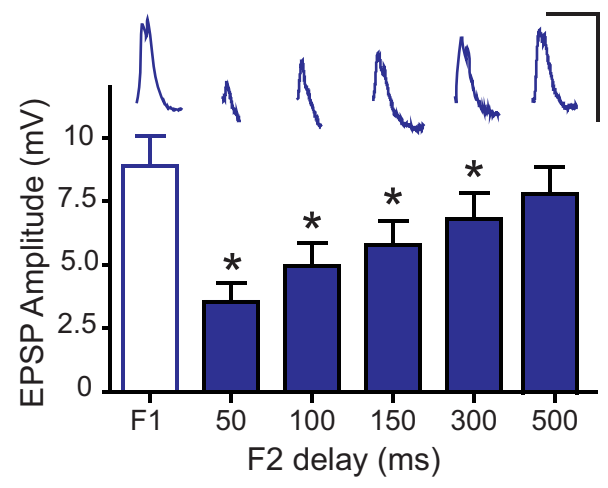

C

E

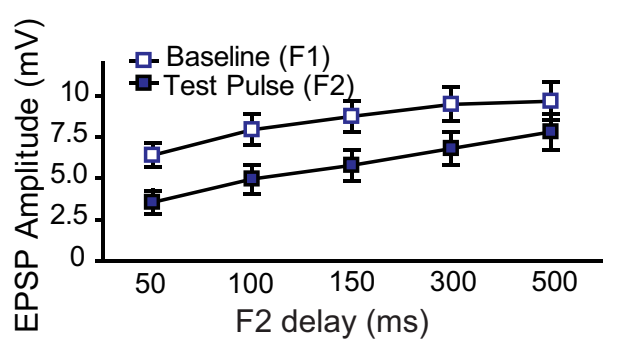

छ

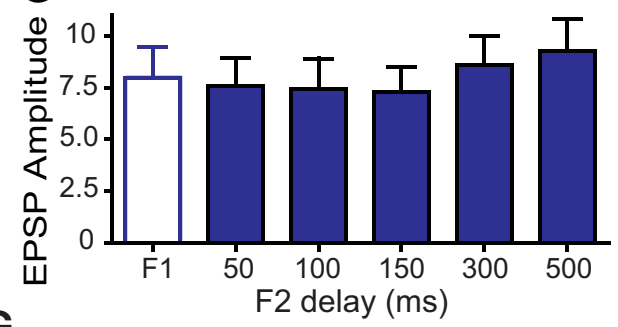

G

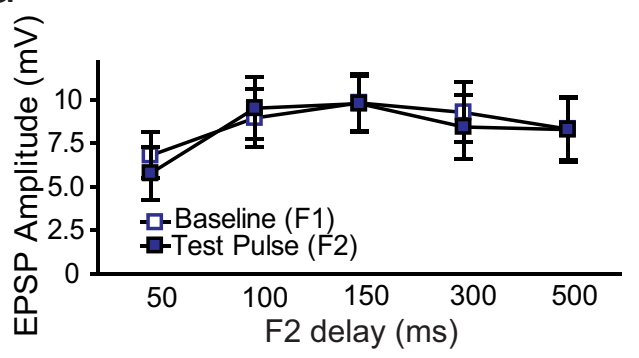

Figure 2. BLA train stimulation evokes heterosynaptic suppression of fornix-evoked synaptic responses in a time-dependent manner. $\boldsymbol{A}$, Top, Stimulation protocol used to study heterosynaptic interactions between BLA and HP inputs to the PFC. Bottom, Overlaid traces from consecutive sweeps of BLA-evoked heterosynaptic suppression of F2 synaptic responses relative to F1 in two representative PFC pyramidal neurons. Blue represents average baseline (F1) and test (F2) fornix-evoked responses. Red represents average responses during BLA train stimulation. The green dashed line indicates the mean baseline $V_{\mathrm{m}}$. Top traces, F2 responses are suppressed well beyond the BLA train and even at potentials more hyperpolarized than baseline. Bottom traces, BLA-evoked heterosynaptic suppression of $F 2$ synaptic responses relative to $F 1$ in a PFC pyramidal neuron where $V_{m}$ during $F 1$ was similar to $V_{m}$ during $F 2$. B, Amplitude of fornix-evoked EPSPs at baseline (F1) and different test pulses (F2) at various delays ( $n=16$ cells from 14 rats). ${ }^{*}$ Significant difference from F1. Average $F 1$ and $F 2$ responses from neuron 1 of Figure $1 A$ are inset above their corresponding column. C, Amplitude of fornix-evoked EPSPs at baseline (F1) and different test pulses (F2) at various delays when F1 and F2 responses were triggered at a similar membrane potential. $\boldsymbol{D}$, Top, Stimulation protocol where the BLA train was omitted ( $n=8$ cells from 8 rats). Bottom, Overlaid traces from consecutive sweeps showing that $F 1$ synaptic responses were similar to $F 2$ responses at all delays. $\boldsymbol{E}$, Amplitude of fornix-evoked EPSPs at baseline (F1) and different test pulses (F2) at various delays. $F$, Top, Stimulation protocol examining the effects of single-pulse BLA stimulation on subsequent $F 2$ responses. Bottom, Overlaid traces from consecutive sweeps demonstrating that single-pulse BLA stimulation does not modify F2 synaptic responses relative to F1. G, Amplitude of fornix-evoked EPSPs at baseline (F1) and different test pulses (F2) at various delays ( $n=10$ cells from 9 rats). In this and subsequent figures, data represent mean \pm SEM. Calibration: $100 \mathrm{ms,}$ $10 \mathrm{mV}$.

ments but with baseline and test pulses applied to the BLA (BLA1 and BLA2) and conditioning pulse train or single pulse applied to the fornix $\left(\mathrm{F}_{\text {train }}\right.$ or $\left.\mathrm{F}_{\text {single }}\right)$.

Optogenetics. Rats were anesthetized with $1-2 \%$ isoflurane. An adenoassociated virus expressing channelrhodopsin-2/YFP under the control of the CaMKII promoter was microinjected into the left ventral HP at two sites (anteroposterior: $-5.6 \mathrm{~mm}$; mediolateral: $5.0 \mathrm{~mm}$; dorsoventral: $-6.5 \mathrm{~mm}$ and $-5.0 \mathrm{~mm}$ from dura; $0.5 \mu \mathrm{l}$ per site). Electrophysiological experiments were conducted 6-8 weeks after viral infection. Instead of a stimulating electrode, an optical fiber $(200 \mu \mathrm{m})$ coupled to a laser diode (473 nm emission; Thor Laboratories) was inserted into the fornix to optically activate transfected ventral HP fibers. Optical stimu- 
lation consisted of a pulse of blue light $(8-15$ $\mathrm{mW}$ ) with durations of 1-5 ms.

Histology. Upon completion of stimulation protocols, Neurobiotin was ejected into cells using a $2 \mathrm{~Hz}$ current pulse ( $100 \mathrm{~ms} ; 0.2-1.2 \mathrm{nA}$ ) through the recoding electrode for 5-20 min. When cells were filled with Neurobiotin, rats were given an overdose of chloral hydrate and transcardially perfused with cold saline followed by $4 \%$ PFA. Brains were postfixed overnight in 4\% PFA, rinsed in PBS, and switched to $30 \%$ sucrose with azide. Sections (40-50 $\mu \mathrm{m})$ were obtained using a freezing microtome. Neurobiotin was visualized using Vectastain Elite $\mathrm{ABC}$ reagent (Vector Laboratories), followed by Fast-DAB (Sigma-Aldrich). Section were Nissl-stained, coverslipped, and examined under a microscope to verify stimulating electrode placements and recovered cell or recording microelectrode track placement (Fig. 1).

Statistical analysis. Heterosynaptic suppression data were analyzed using repeatedmeasures ANOVA with F2 or BLA2 delay as a within-subjects factor. Heterosynaptic suppression data where $\mathrm{F} 1$ and F2 membrane potential was matched was analyzed using repeated-measures ANOVA with both F2, AP2, or BLA2 delay and pulse type (i.e., F1 vs F2) as within-subject factors. Frequencydependent effects of BLA pulse train stimulation were analyzed using repeated-measures ANOVA with both F2 delay and frequency (10, 20 , and $50 \mathrm{~Hz}$ ) as within-subject factors. Post hoc tests were conducted using paired $t$ test or Fisher's least significant difference. Correlations between heterosynaptic suppression and membrane potential difference or depth from cortical surface were determined using linear regression.

\section{Results}

Heterosynaptic suppression of hippocampal inputs to the PFC by BLA train stimulation

Train BLA stimulation attenuated HPevoked EPSPs. A test pulse (F2) to the fornix, the fiber bundle carrying hippocampal fibers innervating the forebrain, was delivered at varying delays after a BLA pulse train ( 5 pulses at $50 \mathrm{~Hz}$; Fig. 2A). BLA train stimulation decreased F2 EPSP amplitude relative to a baseline pulse (F1) in a time-dependent manner in all recorded cells (Fig. $2 A, B ; n=16$ cells from 14 rats). Repeated-measures ANOVA revealed an effect of pulse delay (time between F2 and the last BLA train pulse; $F_{(5,125)}=39.098$; $p<0.0001)$. Post hoc analysis revealed that BLA train stimulation decreased F2 responses relative to $\mathrm{F} 1$ at all intervals $(p<0.05)$, but shorter intervals displayed more robust heterosynaptic suppression than longer delays. A subset of cells showed heterosynaptic suppression lasting $500 \mathrm{~ms}$ or more.

To determine whether BLA-evoked depolarization of PFC pyramidal neurons contributed to heterosynaptic suppression, we restricted our analysis to sweeps in which membrane potential during F1 was similar to that of F2 by taking advantage of the

A
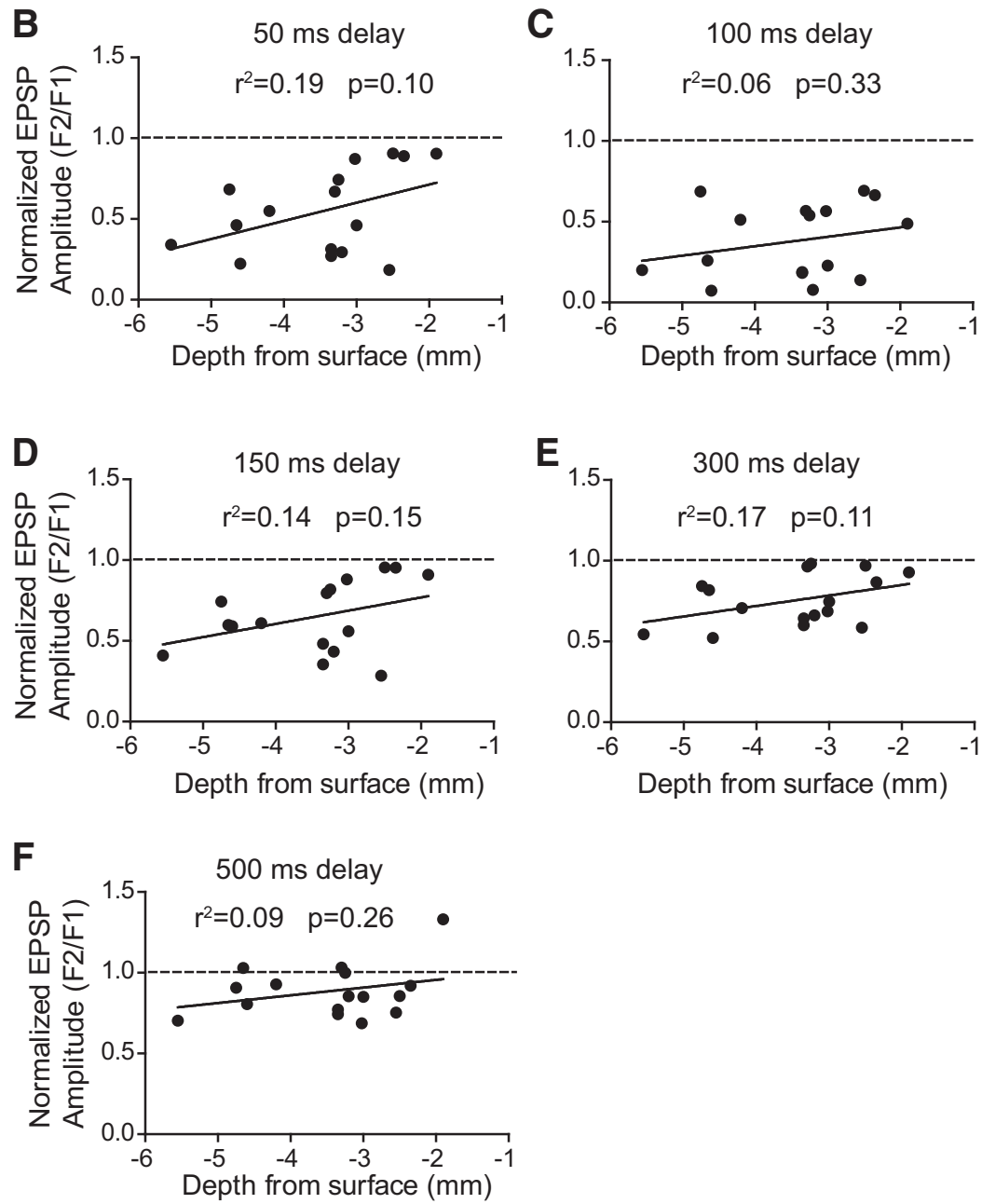

Figure 3. Dorsal and ventral medial PFC sites exhibit similar BLA-evoked heterosynaptic suppression of HP inputs. $\boldsymbol{A}$, Normalized amplitude of fornix-evoked EPSPS (F2/F1) at various delays in cells recorded in the prelimbic ( $n=11$ from 9 rats) and infralimbic cortex ( $n=5$ cells from 5 rats). $\boldsymbol{B}-\boldsymbol{F}$, Relationship between normalized amplitude of fornix-evoked EPSPs (F2/F1) at various delays after BLA train and depth from surface where the medial PFC pyramidal neuron was recorded and respective $r^{2}$ and $p$ values for each regression analysis.

membrane potential alternation between down and up states or by depolarizing the neuron with intracellular injection of positive current. BLA-evoked heterosynaptic suppression was present even when $\mathrm{F} 2$ and $\mathrm{F} 1$ were measured at similar membrane potentials (Fig. 2C). In these cases, repeated-measures ANOVA revealed a delay $\times$ pulse interaction $\left(F_{(4,60)}=23.44 ; p<0.001\right)$. Post hoc analysis revealed a significant decrease in F2 EPSP amplitude relative to F1 at all delays. The lack of dependence of suppression on membrane potential differences at the time F1 and F2 were delivered is also supported by lack of correlation 
A
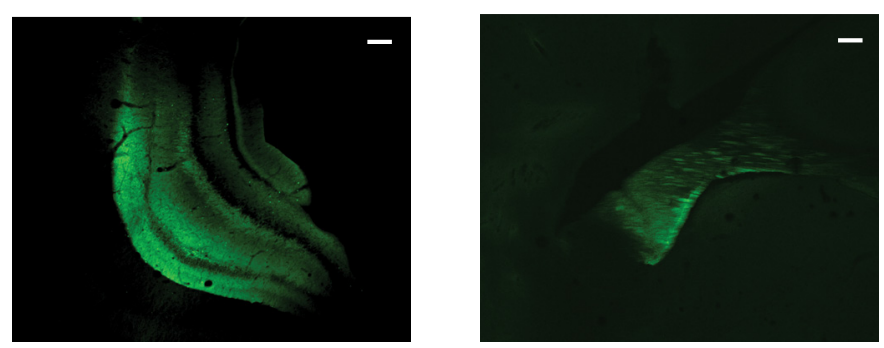

B

F1
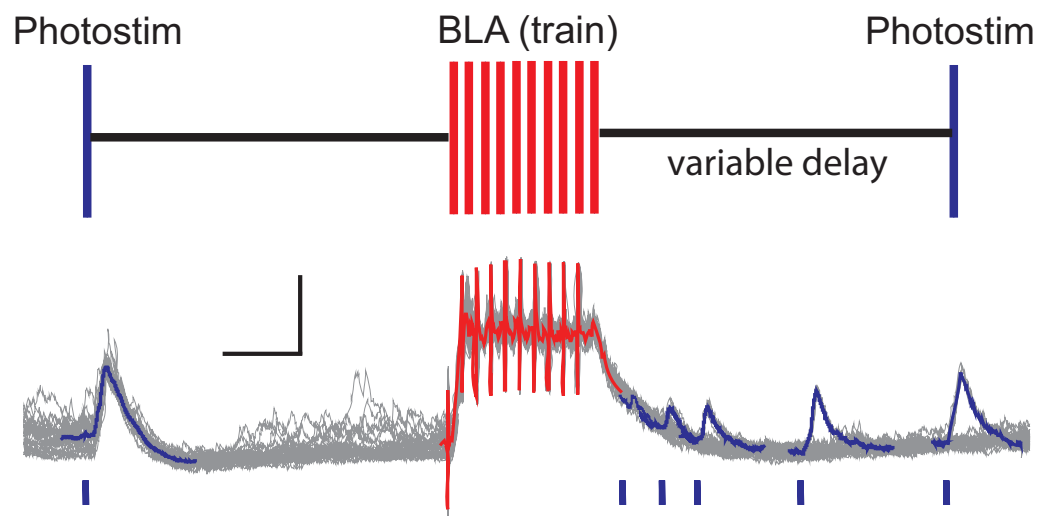

C

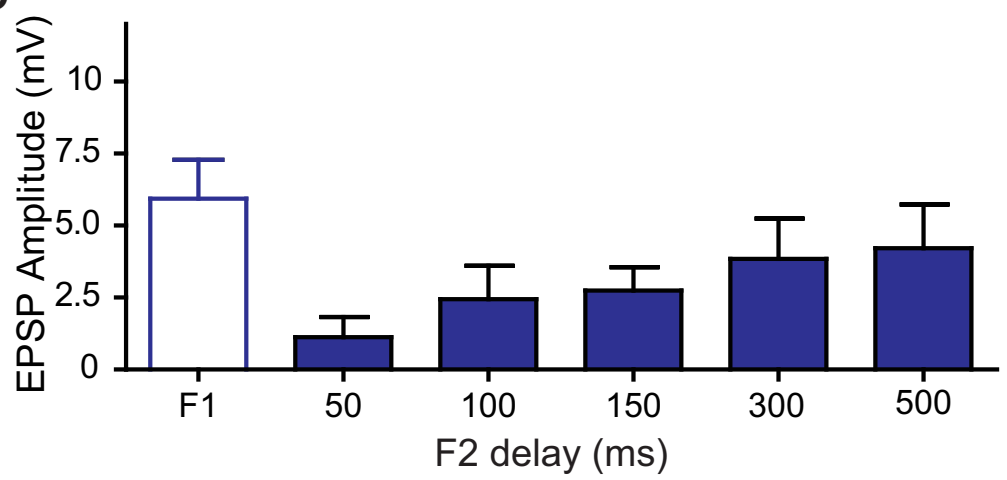

D

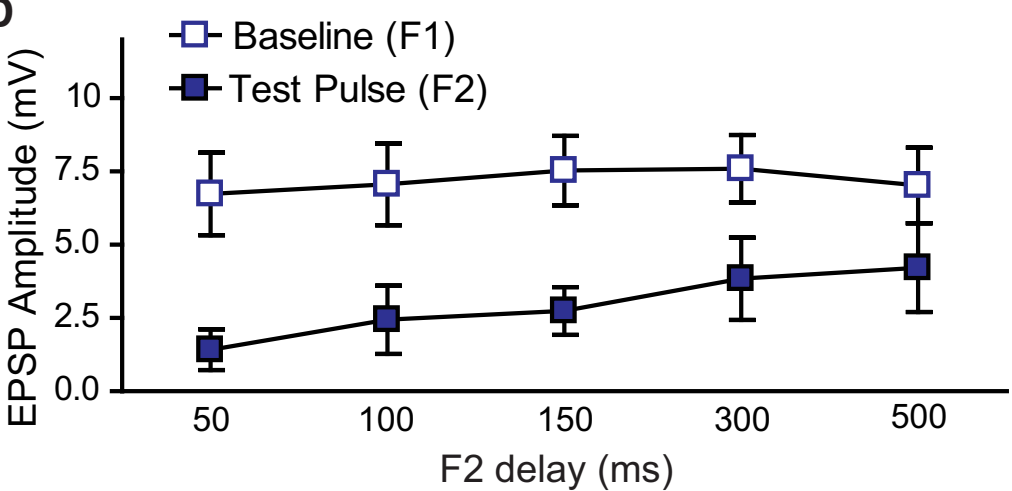

Figure 4. BLA-evoked heterosynaptic suppression of optically evoked fornix responses. $A$, Left, Site of injection into the ventral HP of adenoassociated virus encoding ChR2-YFP. Right, Expression of ChR2 limited to the fornix. Note trackfrom optical fiber placement above the fornix and the BLA-stimulating electrode to the left. B, Top, Stimulation protocol used to study heterosynaptic interactions between BLA and HP inputs to the PFC. Bottom, Overlaid traces showing heterosynaptic suppression of optically evoked hippocampal EPSPS. Blue represents average baseline (F1) and test (F2) hippocampal EPSPS. Red represents average response during BLA train stimulation. C, D, Mean EPSP amplitude of hippocampal-evoked responses at baseline (F1) and different test pulses (F2) delivered at various delays ( $n=7$ cells from 6 rats). Calibration: $100 \mathrm{~ms}, 10 \mathrm{mV}$. between the magnitude of suppression (measured as F2/F1) and the change in membrane potential (measured as $\mathrm{F} 1 V_{\mathrm{m}}$ $\left.-\mathrm{F} 2 V_{\mathrm{m}}\right)$. The correlation values were $p=$ 0.17 for $50 \mathrm{~ms}, p=0.39$ for $100 \mathrm{~ms}, p=$ 0.92 for $150 \mathrm{~ms}, p=0.26$ for $300 \mathrm{~ms}$, and $p=0.83$ for $500 \mathrm{~ms}$ intervals. Moreover, heterosynaptic suppression was still present in neurons in which F2 occurred at more negative membrane potentials than F1, which occurred with longer delays (Fig. 2A,C). These results indicate that BLA train stimulation elicits heterosynaptic suppression of hippocampal afferents to the PFC during a window lasting up to several hundred milliseconds.

It is possible that attenuation of $\mathrm{F} 2$ EPSP amplitude may be due to short-term depression induced by the relatively short F1-F2 interval (730-1180 ms). We assessed this possibility using the same stimulation protocol as described above but omitting the BLA train. We did not observe a significant difference between F1 and $\mathrm{F} 2$ responses at any delay (Fig. $2 D, E$; $\left.F_{(1,35)}=0.02 ; p=0.88\right)$, suggesting that BLA stimulation is required to elicit heterosynaptic suppression ( $n=8$ cells from 8 rats). We have previously demonstrated that single-pulse BLA stimulation drives PFC interneurons and produces inhibitory responses associated with loss of the up states for 100-600 ms (Dilgen et al., 2013). To determine whether single-pulse BLA stimulation was sufficient to evoke heterosynaptic suppression of HP inputs, we repeated experiments using a single BLA pulse. BLA-evoked heterosynaptic suppression of F2 EPSP amplitude was not observed with BLA single-pulse stimulation (Fig. $2 F, G ; n=10$ cells from 9 rats). ANOVA revealed a delay $\times$ pulse interaction $\left(F_{(4,36)}=2.917 ; p=0.035\right)$. However, post hoc analysis failed to reveal any significant difference between $\mathrm{F} 1$ and F2 at any of the time points. These results suggest that train BLA stimulation is required to induce heterosynaptic suppression.

\section{BLA-evoked heterosynaptic} suppression does not differ between dorsal and ventral medial PFC

As dorsal (prelimbic) and ventral (infralimbic) medial PFC exert opposing influences on behavior measures, we compared heterosynaptic suppression in pyramidal neurons in the prelimbic and infralimbic cortex. Heterosynaptic suppression did not differ at any delays between prelimbic and infralimbic cortex (Fig. $3 A ; F_{(1,56)}=0.26 ; p=0.62$ ). Furthermore, regression analysis of the relation- 
ship between heterosynaptic suppression (F2/F1) and depth from cortical surface did not reveal a significant correlation between these two measures (Fig. 3B-F). Therefore, heterosynaptic suppression is uniform across both dorsal and ventral sites within the medial PFC.

\section{BLA-evoked heterosynaptic suppression selectively inhibits hippocampal inputs}

Electrical fornix stimulation could activate nearby nuclei (thalamus) or fibers of passage. To determine the hippocampal specificity of BLA-evoked suppression of responses to fornix electrical stimulation, we used optogenetics. We injected a subset of rats with adeno-associated virus expressing channelrhodopsin-2 (ChR2)YFP under control of the CaMKII promoter into the HP (Fig. 4A). ChR2 was expressed in the fornix at the same site that was targeted for electrical stimulation (Fig. 4A). Optical stimulation with blue light at that site evoked EPSPs similar to those evoked by electrical stimulation. Optically evoked fornix responses were suppressed by $50 \mathrm{~Hz}$ BLA train stimulation (Fig. $4 B, C ; n=7$ cells from 6 rats), even when correcting for membrane potential differences between F1 and F2 (Fig. $4 D)$. ANOVA revealed a pulse $\times$ delay interaction $\left(F_{(4,24)}=2.789 ; p=0.049\right)$. Thus, BLA-evoked heterosynaptic suppression of fornix-evoked responses in the PFC is attributable to inhibition of information flow from the ventral HP to the PFC and not to nonspecific activation of other inputs.

\section{BLA-evoked heterosynaptic} suppression of hippocampal-evoked responses is frequency dependent We next sought to determine whether the frequency of the BLA train determined the degree of heterosynaptic suppression of optically and electrically evoked fornix responses. Using the stimulation protocols described above, but with varied frequency of BLA pulse trains $(10,20$, or 50 $\mathrm{Hz}$; Fig. $5 A$ ), we found that normalized BLA2 EPSP amplitude was temporally attenuated in a frequency-dependent manner (Fig. $5 A, B ; n=5$ cells from 4 rats). Repeated-measures ANOVA revealed a delay $\times$ frequency interaction $\left(F_{(8,32)}=2.789 ; p=\right.$ $0.018)$. These results demonstrate that heterosynaptic suppression becomes more robust and longer-lasting with increasing BLA pulse train frequency.

BLA train stimulation produces heterosynaptic suppression of inputs from an associative temporal cortex

To explore whether BLA-evoked heterosynaptic suppression is restricted to HP inputs, we assessed the effects of BLA train stimulation on responses evoked by electrical stimulation of the AP

B
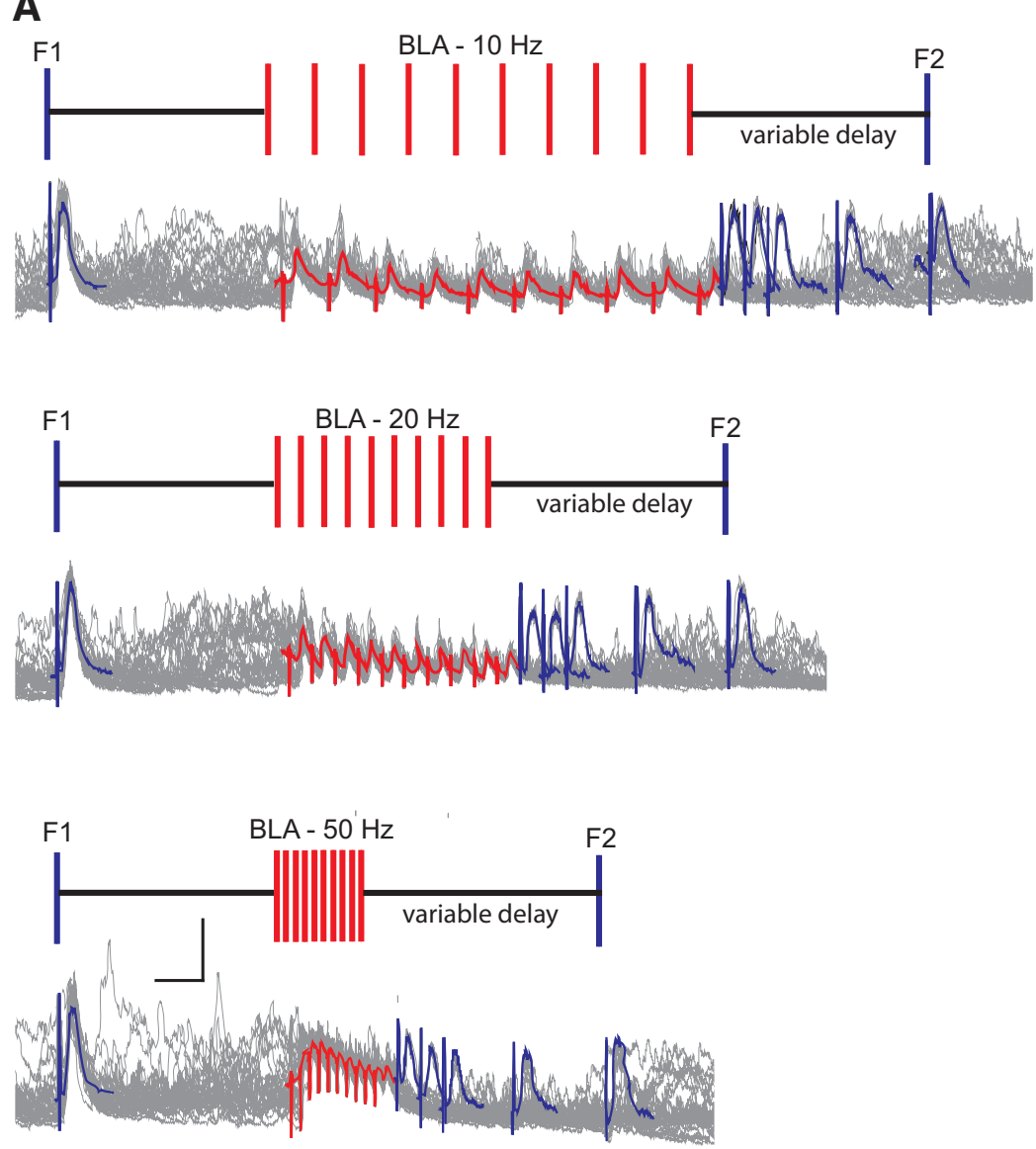

Figure 5. BLA-evoked heterosynaptic suppression of fornix-evoked responses is frequency dependent. A, Stimulation protocols depicted above overlaid traces of heterosynaptic suppression evoked by BLA train stimulation at variable frequencies $(10,20$, and $50 \mathrm{~Hz})$ in a representative neuron. Blue represents average baseline (F1) and test (F2) fornix-evoked responses. Red represents average response during BLA train stimulation. $\boldsymbol{B}$, Mean normalized EPSP amplitude (F2 amplitude/F1 amplitude) at various delays after the last pulse of a BLA train delivered at variable frequencies ( $n=5$ cells from 4 rats). Calibration: $100 \mathrm{~ms}, 10 \mathrm{mV}$.

cortex. The AP is an associative temporal cortical region considered part of the entorhinal cortex that projects to the medial PFC (Condé et al., 1995; Shammah-Lagnado and Santiago, 1999). Single-pulse AP stimulation evoked short-latency monosynaptic EPSPs in PFC neurons similar to those evoked by BLA and fornix stimulation (Fig. 6A), consistent with a direct innervation of PFC neurons by the AP. BLA train stimulation decreased AP-evoked synaptic responses in a time-dependent manner (Fig. $6 B ; n=10$ cells from 8 rats). ANOVA of AP1 and AP2 EPSP amplitudes revealed a delay $\times$ pulse interaction $\left(F_{(1,9)}=5.254 ; p=0.048\right)$. Thus, BLA-mediated heterosynaptic suppression is not limited to $\mathrm{HP}$ afferents to the PFC but is also present in PFC afferents from other cortical structures. 
A

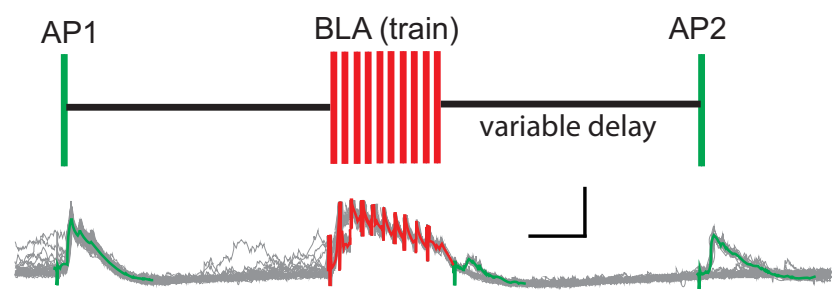

B

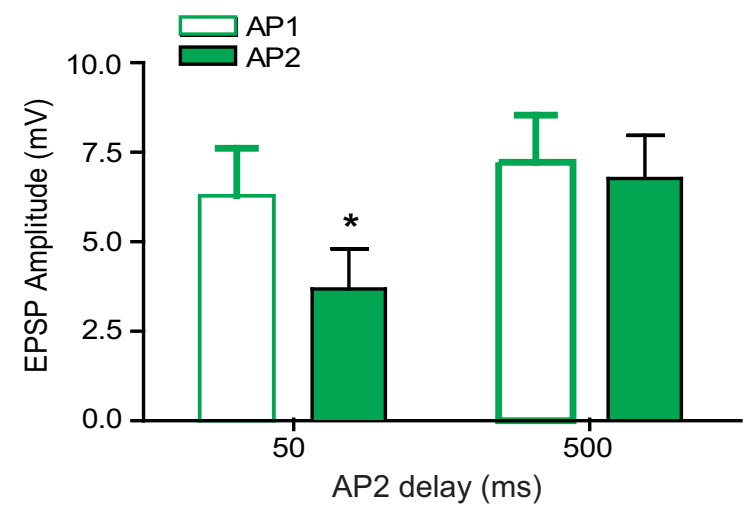

Figure 6. BLA-evoked heterosynaptic suppression is not unique to the hippocampal-PFC pathway. $\boldsymbol{A}$, Stimulation protocols depicted above overlaid traces of heterosynaptic suppression evoked by BLA train stimulation in a representative neuron. Green represents average baseline (APiri1) and test (APiri2) AP cortex (APiri)-evoked responses. Red represents average response during BLA train stimulation. $B$, Mean APiri-evoked EPSP amplitude at a short (50 ms) and long (500 ms) delay after the last pulse of a BLA train is delivered ( $n=10$ cells from 8 rats). ${ }^{*} p<0.05$. Calibration: $100 \mathrm{~ms}, 10 \mathrm{mV}$.

\section{Stimulation of hippocampal inputs to the PFC does not modify BLA-evoked responses in the PFC}

HP stimulation also produces hyperpolarizing responses and inhibits PFC pyramidal neuron activity (Dégenètais et al., 2003; Ishikawa and Nakamura, 2003; Tierney et al., 2004) and, as such, may be able to produce heterosynaptic suppression of the BLAPFC pathway. Stimulation protocols were conducted wherein baseline (BLA1) and test pulses (BLA2) were applied to the BLA while a conditioning pulse train stimulation was applied to the fornix (Fig. $7 A, B$ ). Fornix train stimulation failed to produce heterosynaptic suppression of BLA2 responses relative to BLA1 responses ( $n=8$ cells from 8 rats). Although repeated-measures ANOVA showed a main effect of delay when matching membrane potential $\left(F_{(4,7)}=8.832 ; p<0.001\right)$, there were no BLA1 - BLA2 differences $\left(F_{(1,28)}=1.931 ; p=0.207\right)$. The lack of an effect of fornix stimulation was not due to differential responses than those produced by the BLA as fornix train EPSP amplitude was similar to BLA train EPSP amplitude (data not shown). Furthermore, single-pulse fornix stimulation did not modify BLA2 EPSP amplitude relative to BLA1 at any delay tested (Fig. $7 C, D ; n=8$ cells from 7 rats). ANOVA revealed a main effect of delay $\left(F_{(4,28)}=\right.$ $5.394 ; p=0.002)$, but no effect of pulse type $\left(F_{(1,7)}=1.132 ; p=\right.$ $0.323)$. Although single-pulse fornix stimulation produced hyperpolarizing and inhibitory responses in single-unit activity, it did not attenuate BLA afferents to the PFC. It is possible that short-term facilitation in BLA-evoked responses from repeated BLA stimulation could potentially be masking any heterosynaptic suppressive effects produced by fornix stimulation. However, BLA1 EPSP amplitude did not differ from that of BLA2 when fornix stimulation was omitted (Fig. $7 E, F ; F_{(5,40)}=1.270 ; p=$ 0.296; $n=9$ cells from 9 rats). Collectively, these results suggest that heterosynaptic interactions between the BLA and HP are unidirectional, with BLA suppressing HP inputs, but not vice versa.

\section{Discussion}

Trains of stimuli to the BLA suppressed EPSPs evoked in PFC neurons by fornix stimulation. Heterosynaptic suppression was strongest at shorter delays after the BLA train. BLA-evoked heterosynaptic suppression of HP inputs was not due to membrane potential changes induced by the BLA train and required trains of stimuli, as it was not elicited by paired fornix stimulation in the absence of a BLA pulse train or by single-pulse BLA stimulation. BLA-evoked heterosynaptic suppression did not differ between dorsal (prelimbic) and ventral (infralimbic) regions of the medial PFC. Moreover, BLA-evoked heterosynaptic suppression was frequency-dependent, with more robust and prolonged suppression observed with higher BLA train frequency stimulation. Heterosynaptic suppression of fornix-evoked responses was due to specific action on HP afferents in the PFC, as suppression of EPSPs evoked by optical fornix stimulation was present in animals expressing ChR2 in the ventral HP. We observed heterosynaptic suppression of AP inputs to the PFC, suggesting that other limbic cortical signals are also shunted by BLA inputs. Heterosynaptic suppression was unidirectional and not evoked by fornix train stimulation, suggesting that BLA inputs are distinct from HP inputs in their ability to produce heterosynaptic suppression. Collectively, these results provide a framework whereby BLA activity can interfere with communication between the HP and PFC.

Heterosynaptic suppression was most effective during a short window after BLA stimulation and depended on the intensity of activation. This profile is reminiscent of what is observed during anxiety and stress in humans, conditions in which amygdala activation is inversely correlated to PFC activity (Kim et al., 2011a; Townsend and Altshuler, 2012; Veer et al., 2012). Heterosynaptic suppression was not observed after BLA single-pulse stimulation, suggesting that strong BLA activation is required for heterosynaptic suppression at the delays tested. BLA and HP interactions in the medial PFC have previously been investigated using extracellular single-unit recordings assessing probability of evoked action potential firing with inconsistent results. Single-pulse BLA stimulation was shown to facilitate HP-evoked spiking at short intervals after BLA pulse $(<20 \mathrm{~ms})$ and/or inhibit HP-evoked action potentials in the PFC at intervals longer than $20 \mathrm{~ms}$ in one study (Ishikawa and Nakamura, 2003), and the opposite pattern in another (Esmaeili and Grace, 2013). This discrepancy may be due to a diverse set of experimental conditions that can affect input integration and eventually spiking output. Here, we restricted our assessment to measures of inputs to PFC pyramidal neurons: the amplitude of EPSPs. Although inputs can be modulated by these interactions, additional postsynaptic factors may play a role in further affecting action potential firing.

The mechanisms responsible for heterosynaptic suppression of HP inputs to the PFC are not clear. BLA stimulation inhibits the majority of PFC pyramidal neurons (Pérez-Jaranay and Vives, 1991; Ishikawa and Nakamura, 2003; Floresco and Tse, 2007; Dilgen et al., 2013) via recruitment of local-circuit fastspiking interneurons. Therefore, it is possible that sustained recruitment of PFC interneurons by BLA train stimulation may play a role in shunting incoming HP EPSPs. We previously demonstrated that GABA-A receptor signaling mediates a shortlatency inhibitory effect on single-unit activity (lasting $\sim 50 \mathrm{~ms}$ ) 
A

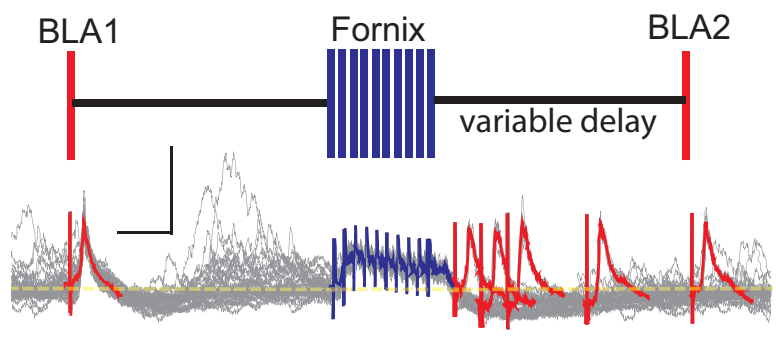

C

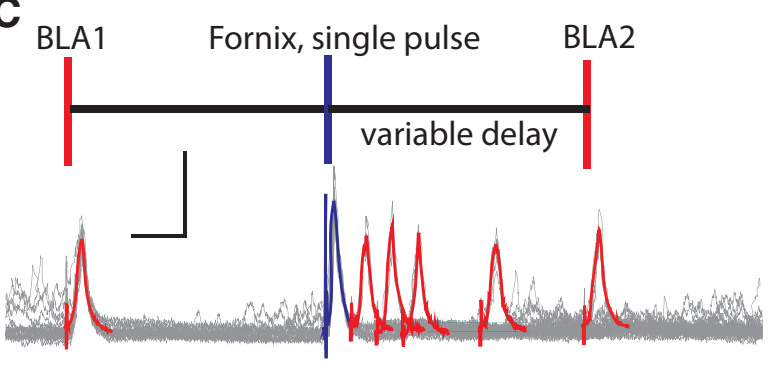

E

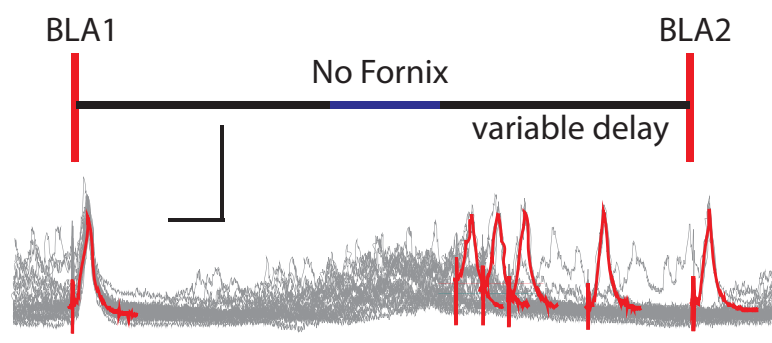

B
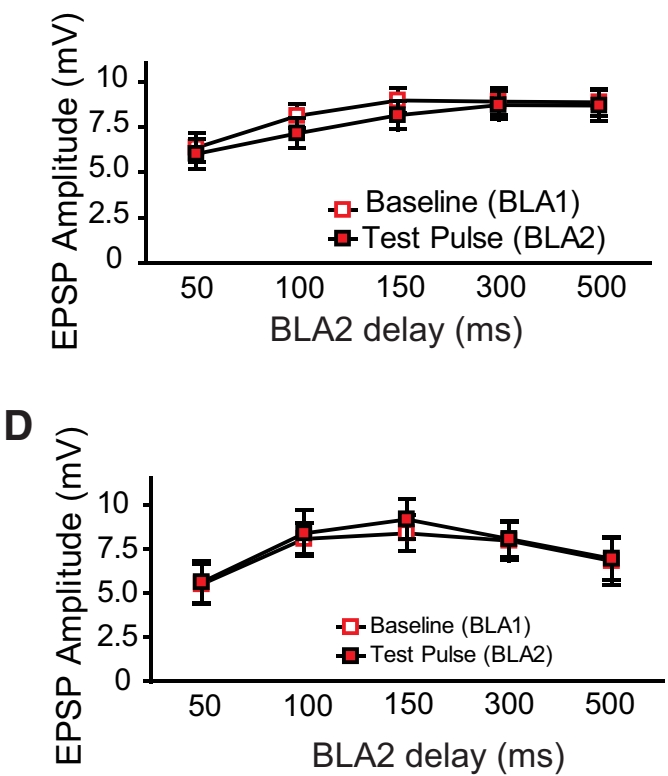

F

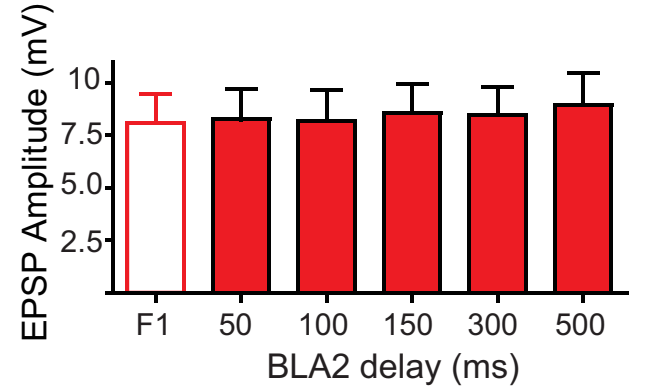

Figure 7. Fornix train stimulation does not induce heterosynaptic suppression of BLA-evoked synaptic responses. $A$, Top, Stimulation protocol. Bottom, Overlaid traces from consecutive sweeps showing the lack of effect of fornix train stimulation on BLA2 synaptic responses relative to BLA1 in a PFC pyramidal neuron. Red represents average baseline (BLA1) and test (BLA2) BLA-evoked responses. Blue represents average response during fornix train stimulation. $B$, Amplitude of BLA-evoked EPSPs at baseline (BLA1) and different test pulses (BLA2) at various delays $(n=8$ cells from 8 rats). C, Top, Stimulation protocol for single-pulse stimulation. Bottom, Overlaid traces from consecutive sweeps demonstrating that single-pulse fornix stimulation does not modify BLA2 synaptic responses relative to BLA1. D, Amplitude of BLA-evoked EPSPs at baseline (BLA1) and different test pulses (BLA2) at various delays ( $n=8$ cells from 7 rats). $\boldsymbol{E}$, Stimulation protocol for no-stimulation experiments. Bottom, Overlaid traces from consecutive sweeps showing that BLA1 synaptic responses were similar to BLA2 responses at all delays when the BLA train was omitted. $F$, Amplitude of BLA-evoked EPSPs at baseline (BLA1) and different test pulses (BLA2) at various delays ( $n=9$ cells from rats). Calibration: $100 \mathrm{~ms}, 10 \mathrm{mV}$.

evoked by single-pulse BLA stimulation, whereas more prolonged hyperpolarizing synaptic responses and inhibition of spiking (lasting $\sim 200 \mathrm{~ms}$ ) are not modified by GABA-A receptor blockade in PFC single units (Dilgen et al., 2013). We also recently reported heterosynaptic suppression in the ventral striatum, which included a component mediated by local GABA-A receptors (Calhoon and O'Donnell, 2013). In that study, blocking GABA-A receptors in the recorded neuron reduced, but did not eliminate, heterosynaptic suppression of HP inputs by PFC stimulation, suggesting that additional mechanisms were involved. It is therefore possible that BLA-evoked heterosynaptic suppression in the PFC is mediated by multiple mechanisms. Short-delay heterosynaptic suppression may be mediated by GABA-A shunting of HP synaptic responses, whereas heterosynaptic suppression expressed at longer delays may be mediated by a slower inhibitory process, such as GABA-B or endocannabinoid signaling. Further work using dual opsin optogenetic approaches using whole-cell electrophysiology in brain slices combined with pharmacological (i.e., blocking postsynaptic GABA-A with picrotoxin in the internal solution or using a GABA-B receptor antagonist) and transgenic (i.e., genetic deletion of presynaptic vs postsynaptic GABA-B receptors) approaches should be used to investigate that factor(s) that mediate heterosynaptic suppression and their impact on afferent inputs to the medial PFC.

Heterosynaptic suppression of temporal cortical inputs to the medial PFC did not differ between dorsal (prelimbic) and ventral (infralimbic) sites. It is well appreciated that dorsal and ventral medial PFC exert opposing effects in aversive and reward learning. For instance, recent work has demonstrated that intermingled BLA neuron populations that project either to the prelimbic or infralimbic PFC are implicated in mediating the expression and extinction of conditioned fear responses, respectively (Senn et al., 2014). BLA stimulation produces proportionally similar excitation and inhibition of both prelimbic medial PFC neurons (Pérez-Jaranay and Vives, 1991; Floresco and Tse, 2007; Dilgen et al., 2013; but see Ishikawa and Nakamura, 2003) and produces similar heterosynaptic suppression of temporal cortical inputs in both prelimbic and infralimbic PFC. Therefore, BLA neurons that project to the PFC may be differentially engaged in conditioned fear by virtue of which medial PFC region they innervate (dorsal vs ventral) and not differential effects on infralimbic and prelimbic cortex neuron activity. It is possible that differential 
recruitment of intermingled BLA neurons projecting either to ventral or dorsal medial PFC partially accounts for divergent functional connectivity between the amygdala and ventral or dorsal BLA during low levels of anxiety compared with that of high levels of anxiety in humans (Kim et al., 2011a,b). Future work should be aimed at determining the influence of selective BLA inputs to either the dorsal and ventral medial PFC on PFC neuron activity and interactions with the hippocampus.

Corticolimbic circuits may shift between bottom-up and topdown inhibition between the BLA and medial PFC depending on situation and contextual information. Signals that cue safety (a neutral stimulus or the periphery of an open field) are associated with increased synchrony in $\theta$ oscillations and spiking between the BLA and medial PFC, which is led by the medial PFC (Likhtik et al., 2014). This is consistent with a role of the medial PFC in attenuating conditioned fear and anxiety responses in the amygdala. Moreover, BLA tended to lead the medial PFC proportionally more in response to cues that predict footshock. Given that BLA neurons can participate in fear expression and extinction depending on the site of medial PFC innervations (Senn et al., 2014), bottom-up regulation of the medial PFC by the BLA may confer information that accentuates and attenuates fear. It is likely that top-down and bottom-up regulation in medial PFC and BLA pathways shifts back and forward depending on perceived signs of threat and safety to engage in an appropriate behavioral response. This is evidenced by differences in medial PFC-BLA neuron activity synchrony that is led by either the BLA or medial PFC depending on whether monkeys can successfully discriminate between reversal learning of conditioned fear and neutral stimuli (Klavir et al., 2013).

HP afferents do not appear to gate integration of BLA-evoked synaptic responses in the PFC. Fornix train stimulation failed to produce heterosynaptic suppression of BLA-evoked responses, suggesting that heterosynaptic suppression by the BLA is unidirectional. This is consistent with a recent study demonstrating that single-pulse HP stimulation fails to modify BLA-evoked spiking of PFC neurons (Esmaeili and Grace, 2013). Inhibition of BLA-evoked spiking by a priming HP pulse has also been previously reported, but this effect lasted $<80 \mathrm{~ms}$ (Ishikawa and $\mathrm{Na}$ kamura, 2003). The HP may play a limited role in gating BLA inputs to the PFC. It is not clear why HP inputs would not be able to suppress BLA inputs, as HP inputs increase PFC interneuron activity and inhibit the activity of PFC neurons (Dégenètais et al., 2003; Tierney et al., 2004) similar to the BLA. HP train stimulation at frequencies similar to those used in the present study do recruit inhibitory components involving GABA-A receptors (Thomases et al., 2013). Therefore, it is likely that the unidirectional gating we observed is not due to differences in the ability of BLA and HP afferents to recruit inhibitory interneurons in PFC networks. It is possible that differential location of BLA and hippocampal synapses onto PFC pyramidal neurons accounts for a lack of hippocampal-evoked heterosynaptic suppression of BLA inputs. Anatomically, both the ventral hippocampus and BLA heavily innervate layers II/III and deep layers of the medial PFC, but innervation does not predict the presence of functional synapses (Little and Carter, 2012). In layer II pyramidal neurons, functional hippocampal synapses with NMDA receptors are found in both proximal and distal dendrites, whereas BLA inputs with functional NMDA receptors are located more proximal relative to hippocampal afferents. If proximal BLA inputs engage a shunting conductance (i.e., a $\mathrm{K}^{+}$conductance) in proximal dendrites that it innervates, that would influence depolarizations traveling from distal to proximal sites. Hippocampal inputs acti- vating similar conductances in distal dendrites would not be expected to influence BLA-evoked depolarizations in proximal dendrites. It is also possible that HP-mediated heterosynaptic suppression exists, but it may be dependent on the state of the network. HP inactivation did not influence encoding of fear in the PFC in fear-conditioned rats but did in rats that had undergone extinction (Sotres-Bayon et al., 2012). This notion is also consistent with a recent study indicating that the HP can exert an inhibitory influence on BLA-evoked extracellular responses using high-frequency stimulation protocols that elicit long-term changes in synaptic efficacy (Thomases et al., 2014). Moreover, in a developmental animal model of schizophrenia, BLA-evoked heterosynaptic suppression is lost, whereas HP inhibition of BLA-evoked orthodromic spikes is gained (Esmaeili and Grace, 2013).

Heterosynaptic suppression may serve to restrict activated PFC neural ensembles to those relevant to the behavioral state driving BLA activity. Furthermore, our findings that heterosynaptic suppression requires trains of pulses to the BLA and is dependent on the frequency of BLA train stimulation suggest that BLA activity may be able to inversely scale PFC responsiveness to HP and cortical inputs that drive PFC network activity, and the BLA would most effectively gate HP information flow into the PFC in situations where the BLA would be strongly activated. By inhibiting synaptic HP responses in the PFC, the BLA may also influence HP entrainment of $\theta$ oscillations in the PFC, which are associated with optimal mnemonic processing (Gordon, 2011). BLA stimulation also suppressed AP cortex inputs to the medial PFC, suggesting that BLA is well positioned to regulate the flow of information from other temporal cortical structures. Heterosynaptic suppression of HP and cortical afferents is one mechanism by which the BLA could decrease PFC activation and may provide a basis for "bottom-up" inhibition of PFC activity by the BLA. Under normal resting states, unidirectional heterosynaptic suppression may be the default mode interaction, only overcome by strong HP and temporal cortical activity. This interaction may sharpen BLA-driven PFC neuronal ensembles, in part by decreasing "noise" by heterosynaptic suppression of weak inputs. Dampening HP and other inputs would allow only the strongest HP and cortical inputs, capable of overriding heterosynaptic suppression to influence PFC neural ensembles. BLA activity is necessary for conditioned fear-induced increases in PFC neuron firing (Laviolette et al., 2005; Sotres-Bayon et al., 2012), but BLA activity suppresses the majority of PFC neurons during expression of conditioned fear (Garcia et al., 1999). These findings are consistent with the hypothesis that BLA-mediated PFC inhibition and heterosynaptic suppression may highlight the activity of behaviorally relevant PFC ensembles. Such control of sparse coding in PFC ensembles by the BLA via heterosynaptic suppression would affect PFC-dependent decision-making and cognition. This would be a mechanism by which emotional valence or any other information being processed in the BLA can influence PFC output.

\section{References}

Bechara A, Van Der Linden M (2005) Decision-making and impulse control after frontal lobe injuries. Curr Opin Neurol 18:734-739. CrossRef Medline

Bechara A, Damasio H, Damasio AR (2003) Role of the amygdala in decision-making. Ann N Y Acad Sci 985:356-369. CrossRef Medline

Calhoon GG, O’Donnell P (2013) Closing the gate in the limbic striatum: prefrontal suppression of hippocampal and thalamic inputs. Neuron 78: 181-190. CrossRef Medline

Chudasama Y, Robbins TW (2006) Functions of frontostriatal systems in 
cognition: comparative neuropsychopharmacological studies in rats, monkeys and humans. Biol Psychol 73:19-38. CrossRef Medline

Churchwell JC, Morris AM, Heurtelou NM, Kesner RP (2009) Interactions between the prefrontal cortex and amygdala during delay discounting and reversal. Behav Neurosci 123:1185-1196. CrossRef Medline

Condé F, Maire-Lepoivre E, Audinat E, Crépel F (1995) Afferent connections of the medial frontal cortex of the rat: II. Cortical and subcortical afferents. J Comp Neurol 352:567-593. CrossRef Medline

Dégenètais E, Thierry AM, Glowinski J, Gioanni Y (2003) Synaptic influence of hippocampus on pyramidal cells of the rat prefrontal cortex: an in vivo intracellular recording study. Cereb Cortex 13:782-792. CrossRef Medline

Dilgen J, Tejeda HA, O’Donnell P (2013) Amygdala inputs drive feedforward inhibition in the medial prefrontal cortex. J Neurophysiol 110:221229. CrossRef Medline

Eichenbaum H (2000) A cortical-hippocampal system for declarative memory. Nat Rev Neurosci 1:41-50. CrossRef Medline

Esmaeili B, Grace AA (2013) Afferent drive of medial prefrontal cortex by hippocampus and amygdala is altered in MAM-treated rats: evidence for interneuron dysfunction. Neuropsychopharmacology 38:1871-1880. CrossRef Medline

Floresco SB, Ghods-Sharifi S (2007) Amygdala-prefrontal cortical circuitry regulates effort-based decision making. Cereb Cortex 17:251-260. CrossRef Medline

Floresco SB, Tse MT (2007) Dopaminergic regulation of inhibitory and excitatory transmission in the basolateral amygdala-prefrontal cortical pathway. J Neurosci 27:2045-2057. CrossRef Medline

Garcia R, Vouimba RM, Baudry M, Thompson RF (1999) The amygdala modulates prefrontal cortex activity relative to conditioned fear. Nature 402:294-296. CrossRef Medline

Gordon JA (2011) Oscillations and hippocampal-prefrontal synchrony. Curr Opin Neurobiol 21:486-491. CrossRef Medline

Ishikawa A, Nakamura S (2003) Convergence and interaction of hippocampal and amygdalar projections within the prefrontal cortex in the rat. J Neurosci 23:9987-9995. Medline

Kim MJ, Gee DG, Loucks RA, Davis FC, Whalen PJ (2011a) Anxiety dissociates dorsal and ventral medial prefrontal cortex functional connectivity with the amygdala at rest. Cereb Cortex 21:1667-1673. CrossRef Medline

Kim MJ, Loucks RA, Palmer AL, Brown AC, Solomon KM, Marchante AN, Whalen PJ (2011b) The structural and functional connectivity of the amygdala: from normal emotion to pathological anxiety. Behav Brain Res 223:403-410. CrossRef Medline

Klavir O, Genud-Gabai R, Paz R (2013) Functional connectivity between amygdala and cingulate cortex for adaptive aversive learning. Neuron 80:1290-1300. CrossRef Medline

Laviolette SR, Lipski WJ, Grace AA (2005) A subpopulation of neurons in the medial prefrontal cortex encodes emotional learning with burst and frequency codes through a dopamine D4 receptor-dependent basolateral amygdala input. J Neurosci 25:6066-6075. CrossRef Medline
Likhtik E, Pelletier JG, Paz R, Paré D (2005) Prefrontal control of the amygdala. J Neurosci 25:7429-7437. CrossRef Medline

Likhtik E, Stujenske JM, Topiwala MA, Harris AZ, Gordon JA (2014) Prefrontal entrainment of amygdala activity signals safety in learned fear and innate anxiety. Nat Neurosci 17:106-113. CrossRef Medline

Little JP, Carter AG (2012) Subcellular synaptic connectivity of layer 2 pyramidal neurons in the medial prefrontal cortex. J Neurosci 32:1280812819. CrossRef Medline

Paxinos G, Watson C (1998) The rat brain in stereotaxic coordinates. San Diego: Academic.

Pérez-Jaranay JM, Vives F (1991) Electrophysiological study of the response of medial prefrontal cortex neurons to stimulation of the basolateral nucleus of the amygdala in the rat. Brain Res 564:97-101. CrossRef Medline

Phelps EA, LeDoux JE (2005) Contributions of the amygdala to emotion processing: from animal models to human behavior. Neuron 48:175-187. CrossRef Medline

Rosenkranz JA, Grace AA (2002) Cellular mechanisms of infralimbic and prelimbic prefrontal cortical inhibition and dopaminergic modulation of basolateral amygdala neurons in vivo. J Neurosci 22:324-337. Medline

Senn V, Wolff SB, Herry C, Grenier F, Ehrlich I, Gründemann J, Fadok JP, Müller C, Letzkus JJ, Lüthi A (2014) Long-range connectivity defines behavioral specificity of amygdala neurons. Neuron 81:428-437. CrossRef Medline

Shammah-Lagnado SJ, Santiago AC (1999) Projections of the amygdalopiriform transition area (APir): a PHA-L study in the rat. Ann N Y Acad Sci 877:655-660. CrossRef Medline

Sotres-Bayon F, Sierra-Mercado D, Pardilla-Delgado E, Quirk GJ (2012) Gating of fear in prelimbic cortex by hippocampal and amygdala inputs. Neuron 76:804-812. CrossRef Medline

Sripada RK, King AP, Garfinkel SN, Wang X, Sripada CS, Welsh RC, Liberzon I (2012) Altered resting-state amygdala functional connectivity in men with posttraumatic stress disorder. J Psychiatry Neurosci 37:241-249. CrossRef Medline

Thomases DR, Cass DK, Tseng KY (2013) Periadolescent exposure to the NMDA receptor antagonist MK-801 impairs the functional maturation of local GABAergic circuits in the adult prefrontal cortex. J Neurosci 33:26-34. CrossRef Medline

Thomases DR, Cass DK, Meyer JD, Caballero A, Tseng KY (2014) Early adolescent MK-801 exposure impairs the maturation of ventral hippocampal control of basolateral amygdala drive in the adult prefrontal cortex. J Neurosci 34:9059-9066. CrossRef Medline

Tierney PL, Dégenètais E, Thierry AM, Glowinski J, Gioanni Y (2004) Influence of the hippocampus on interneurons of the rat prefrontal cortex. Eur J Neurosci 20:514-524. CrossRef Medline

Townsend J, Altshuler LL (2012) Emotion processing and regulation in bipolar disorder: a review. Bipolar Disord 14:326-339. CrossRef Medline

Veer IM, Oei NY, Spinhoven P, van Buchem MA, Elzinga BM, Rombouts SA (2012) Endogenous cortisol is associated with functional connectivity between the amygdala and medial prefrontal cortex. Psychoneuroendocrinology 37:1039-1047. CrossRef Medline 